LEADING ARTICLE

\title{
Advances in our understanding of the pathology of chronic intestinal pseudo-obstruction
}

\author{
R De Giorgio, G Sarnelli, R Corinaldesi, V Stanghellini
}

Gut 2004;53:1549-1552. doi: 10.1136/gut.2004.043968

Chronic intestinal pseudo-obstruction (CIP) represents a particularly difficult clinical challenge. It is a rare and highly morbid syndrome characterised by impaired gastrointestinal propulsion together with symptoms and signs of bowel obstruction in the absence of any lesions occluding the gut lumen. CIP can be classified as either "secondary" to a wide array of recognised pathological conditions or "idiopathic" (CIIP). This review will focus on CIIP, and specifically on the underlying pathological abnormalities. Combined clinical and histopathological studies are needed to highlight new perspectives in the understanding and management of chronic intestinal pseudo-obstruction.

See end of article for authors' affiliations

Correspondence to: Dr R De Giorgio, Department of Internal Medicine and

Gastroenterology, St Orsola-Malpighi Hospital, Via Massarenti, 9, I-40138 Bologna, Italy; deg@orsola-malpighi. med.unibo.it

Revised version received 24 June 2004

Accepted for publication

1 July 2004

\section{SUMMARY}

The histopathology of chronic intestinal pseudoobstruction has been frequently reported as a frustrating experience by gastrointestinal pathologists. Renewed interest in gut neuromuscular pathology has been fuelled by the availability of full thickness biopsies obtained with minimally invasive surgical techniques, improvement in tissue preservation, and refinement of a number of morphofunctional techniques. Pathological abnormalities underlying chronic idiopathic intestinal pseudo-obstruction can be classified into three major entities: neuropathies, "mesenchymopathies" (that is, changes in interstitial cells of Cajal network), and myopathies. Inflammatory/immune mediated neuropathies are characterised by either a predominant $\mathrm{T}$ cell (CD4 and CD8 lymphocytes) or eosinophilic infiltrate within the myenteric plexus. Other forms of neuropathies are characterised by loss of neurones together with evidence of neurodegenerative aspects in the absence of an identifiable inflammatory response. Abnormalities of interstitial cells of Cajal and smooth muscle cells may also contribute to gut dysmotility. Combined clinical and histopathological studies will open new perspectives in the understanding and management of chronic intestinal pseudoobstruction.

\section{CHRONIC INTESTINAL PSEUDO- OBSTRUCTION: A GENERAL OVERVIEW ON A RARE DISEASE}

Digestive motility is a highly coordinated process which enables mixing, absorption, and propulsion of the ingesta through the gastrointestinal tract up to expulsion of residues. This function depends on smooth muscle contractility and the related pacemaker activity evoked by the interstitial cells of Cajal (ICCs), both finely tuned by intrinsic (that is, the enteric nervous system (ENS)) and extrinsic (that is, sympathetic and parasympathetic) nerves. ${ }^{12}$ Disturbances in digestive motility can occur as a result of a variety of abnormalities affecting each of these elements (alone or in combination) involved in the physiology of gut motor function. ${ }^{3-7}$

\section{"Intestinal pseudo-obstruction represents a particularly difficult clinical challenge"}

Intestinal pseudo-obstruction represents a particularly difficult clinical challenge. It is a rare and highly morbid syndrome characterised by impaired gastrointestinal propulsion together with symptoms and signs of bowel obstruction in the absence of any lesions occluding the gut lumen. ${ }^{3-5}$ Pseudo-obstructive syndromes may be either acute (due to abdominal surgery, retroperitoneal haemorrhage, spinal or pelvic trauma, myocardial infarction, or hypokalaemia) ${ }^{8}$ or, more commonly, chronic. The latter form (that is, chronic intestinal pseudo-obstruction (CIP)) is an important cause of chronic functional intestinal failure. CIP can be further classified as either "secondary" to a wide array of recognised pathological conditions ${ }^{3-5}$ or "idiopathic" (CIIP). Although familial forms with autosomal dominant or recessive modes of inheritance have been reported,,$^{3-7}$ most cases of CIIP appear to be unrelated to familial clusters and therefore are referred to as sporadic forms. This review will focus on CIIP, and specifically on the underlying pathological abnormalities.

\section{CLINICAL AND DIAGNOSTIC FEATURES}

CIIP patients present with recurrent episodes of abdominal pain, nausea and/or vomiting, and distension and/or bloating mimicking a mechanical sub-occlusion. ${ }^{3-7}$ Diarrhoea and steatorrhoea may occur as a result of bacterial overgrowth of the small intestine. Dysphagia is present in a low percentage of patients with CIIP although it is relatively frequent in those affected by pseudoobstruction secondary to progressive systemic sclerosis. Nausea, vomiting, and weight loss are

Abbreviations: ANNA-1, antineuronal nuclear antibodies; BCL-2, B cell lymphoma-2; CIP, chronic intestinal pseudo-obstruction; CIIP, chronic intestinal idiopathic pseudo-obstruction; ICC, interstitial cells of Cajal; ENS, enteric nervous system 
predominant symptoms when gut dysfunction affects the upper gastrointestinal tract whereas diffuse abdominal pain, severe distension, and constipation are suggestive of an impairment primarily affecting the distal gut. Between subocclusive episodes, patients may be asymptomatic or complain of symptoms of variable intensity. The onset of CIIP is generally insidious (that is, gastrointestinal symptoms of increasing severity may precede the first sub-occlusive episode by many years) or, less frequently, it is abrupt (that is, presenting with a sub-occlusive episode). ${ }^{9}$ Repeated laparotomies, often preceding the correct diagnosis, represent a common finding in the clinical history of these patients.

The diagnosis of CIIP is mainly clinical and confirmed by endoscopic or radiological exclusion of mechanical causes as well as by evidence of air-fluid levels in distended bowel loops..$^{3-9}$ Standard or ambulatory intestinal manometry, although not mandatory in the diagnostic workup of CIIP, may help in differentiating mechanical from functional forms of sub-occlusion. This test also provides information on the origin of the underlying dysmotility, referred to as myogenic or neurogenic depending on the putative abnormality involving the smooth muscle or the innervation (intrinsic or extrinsic). ${ }^{10}$ In neuropathic CIIP, contractions are uncoordinated although they have normal amplitude; conversely, in myopathic forms, intestinal contractions are coordinated but of low amplitude, if detectable. Nevertheless, intestinal manometry does not provide clues to the intimate nature of the neuromuscular impairment. As this information is relevant for diagnosis, prognosis, and management of patients with CIIP, full thickness biopsies have long been proposed for appropriate tissue analysis. However, the potential benefit derived from tissue sampling has been hampered by the common clinical experience that surgery often worsened the underlying dysmotility either directly (by as yet unknown mechanisms) or by increasing the presence of adhesions, thus complicating the clinical picture with an overlapping "mechanical" factor. Laparotomy often resulted in useless resections of dilated bowel loops and tissue analysis often led the pathologists to diagnose an apparently "normal" neuromuscular layer. Lack of a recognised and accepted nosology of enteric neuromuscular disorders may have contributed to unclear histopathological diagnoses.

\section{"The diagnosis of CIIP is mainly clinical and confirmed by endoscopic or radiological exclusion of mechanical causes as well as by evidence of air-fluid levels in distended bowel loops"}

However, during the 1980s, Krishnamurthy and Schuffler ${ }^{11}$ proposed a classification of enteric neuromuscular abnormalities in patients with severe dysmotility, based mainly on histochemical assessment. Although accurately detailed, this classification progressively lost its intrinsic value as a result of the overwhelming knowledge emerging from basic studies on enteric neuromuscular structure and function (for review see Goyal and Hirano, ${ }^{1}$ Wood, ${ }^{2}$ Furness and Costa, ${ }^{12}$ and Gershon and colleagues ${ }^{13}$ ).

The recent advancement provided by minimally invasive approaches, such as laparoscopic surgery, together with the availability of a wide array of molecular and morphofunctional tests to analyse gut tissues have refuelled the interest for full thickness intestinal biopsy in patients with CIIP. So far, there are no definite criteria for establishing which patients with severe dysmotility may benefit from gastrointestinal tissue biopsy. Biopsies may be indicated in patients with severe derangements of gut motility of unknown origin unresponsive to therapy, with suspected mechanical obstruction (not excluded by preoperative tests), or in patients with a permanent catheter for enteral or parenteral nutrition.

\section{CLASSIFICATION AND MECHANISMS OF ENTERIC NEUROMUSCULAR DYSFUNCTION}

Based on tissue examination, CIIP can be classified into three major entities: neuropathies, "mesenchymopathies", and myopathies, depending on the predominant involvement of enteric neurones, ICCs, or smooth muscle cells. ${ }^{5-7}$ Although each of these entities may be responsible for dysmotility, combined forms (for example, neuromyopathies) may also coexist in the same tissue specimen.

\section{Neuropathies}

Damage to the functional and/or structural integrity of the ENS plays a major role in gut dysmotility..$^{3-8}$ This is due to the high morphofunctional complexity of the ENS which is able to control, independently from the central and peripheral nervous systems, virtually all gut functions, including motility. ${ }^{12}{ }^{12} 13$ Neuropathic CIIP can be classified into two major forms: (a) inflammatory neuropathies in which a significant inflammatory/immune response is identified within enteric ganglia and/or nerve processes; and (b) degenerative neuropathies characterised by evidence of neurodegenerative aspects in the absence of an identifiable inflammatory response.

\section{"Damage to the functional and/or structural integrity of the ENS plays a major role in gut dysmotility"}

Inflammatory neuropathies are characterised by a dense infiltrate of lymphocytes and plasma cells involving either of the two major ganglionated plexuses, although mainly the myenteric plexus (that is, myenteric ganglionitis) and axons of the ENS. ${ }^{6714}$ Usually, cases of myenteric ganglionitis are secondary to several diseases, including paraneoplastic (for example, small cell carcinoma, carcinoid, neuroblastoma, and thymoma), infectious (for example, Chagas' disease), neurological (for example, encephalomyeloneuropathy), connective tissue (for example, scleroderma), and inflammatory bowel disorders (for review see De Giorgio and colleagues ${ }^{14}$ ). Nevertheless, some cases may be idiopathic in origin. ${ }^{15-17}$ Immunohistopathological analysis shows an immune infiltrate composed of predominant CD4 ( $\mathrm{T}$ helper) and CD8 ( $\mathrm{T}$ suppressor) lymphocytes (fig 1 ) which can be identified in idiopathic and secondary forms of myenteric ganglionitis. Lymphocytic myenteric ganglionitis is often associated with neuronal changes indicative of degeneration and loss up to complete ganglion cell depletion occurring in the most severe forms (a feature referred to as acquired aganglionosis). ${ }^{15}{ }^{17}$ In addition to cell mediated immune injury, patients with lymphocytic myenteric ganglionitis develop a humoral response characterised by antineuronal antibodies, namely antinuclear neuronal antibodies (ANNA-1) or anti-Hu (from the name of the molecular target recognised by these autoantibodies). ${ }^{18}$ Detection of ANNA-l/anti-Hu antibodies in the serum of patients with idiopathic myenteric ganglionitis is useful for diagnosis and helps to establish the rationale for appropriate immunosuppressive treatment. By contrast, recent data showed a predominant eosinophilic infiltrate in the myenteric plexus of paediatric cases of CIIP which does not appear to be associated with overt neurodegeneration. ${ }^{19}$

Degenerative non-inflammatory neuropathies may occur as a result of endogenous and/or exogenous noxae leading to damage and loss of enteric neurones. Typical neuropathological findings include marked reduction of intramural (especially myenteric) neurones associated with swollen cell bodies and processes, fragmentation and loss of axons, and 


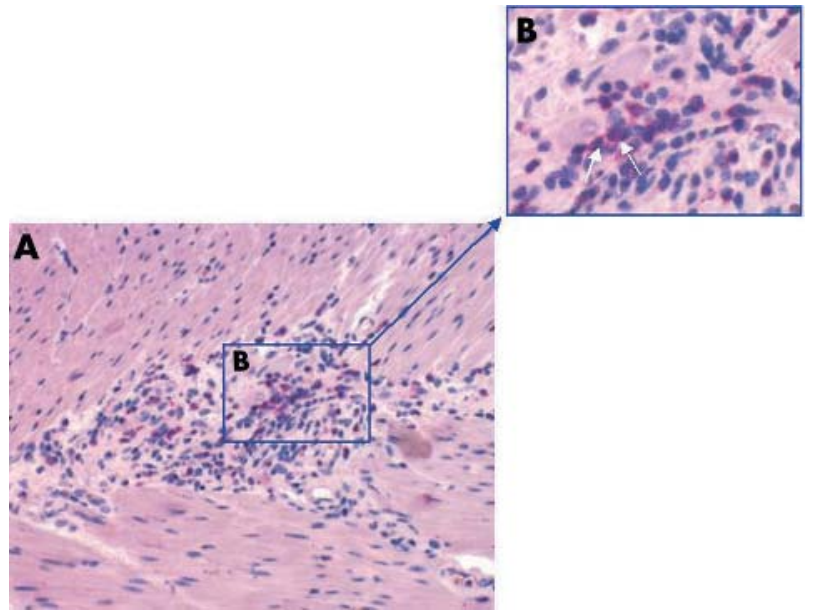

Figure 1 Representative photomicrograph illustrating a case of idiopathic lymphocytic myenteric ganglionitis. The immune infiltrate is localised within the myenteric plexus of the small bowel of a patient with chronic intestinal idiopathic pseudo-obstruction. The positive immunolabelling (light red/pink colour) identifies CD8 T lymphocytes (A). The inset selected (B) is a higher magnification of (A) showing the close proximity between CD8 positive lymphocytes and myenteric neurones (arrows). Alkaline phosphatase antialkaline phosphatase immunohistochemical technique. Original magnifications: $80 \times, A$; $160 \times$, B.

proliferation of glial cells. ${ }^{6711}$ Remaining neurones may be enlarged with thick clubbed processes and associated with an increased number of Schwann cells and hypertrophy of the muscularis propria. Although several studies have focused on neurotransmitter disorders of the ENS in colonic inertia, only a few have been performed in patients with degenerative neuropathy underlying CIIP.

"Enteric neurones of patients with CIIP display reduced expression of the protein encoded by BCL-2 ( $B$ cell lymphoma-2), a gene related to one of the intracellular pathways leading to programmed cell death"

Possible pathogenetic mechanisms involved in central nervous system neurodegenerative disorders have been assumed to occur in enteric neuropathies due to the high degree of similarity shared by central and enteric neurones. ${ }^{13}$ Neurodegenerative mechanisms include altered calcium signalling, mitochondrial dysfunction, and production of free radicals..$^{20}$ Preliminary data have demonstrated that enteric neurones of patients with CIIP display reduced expression of the protein encoded by BCL-2 (B cell lymphoma-2), a gene related to one of the intracellular pathways leading to programmed cell death, ${ }^{21}$ and this decreased BCL-2 expression was associated with enhanced activation of neuronal programmed cell death/apoptosis in CIIP tissues. ${ }^{22}$

\section{ICC abnormalities}

Alterations in the ICC network have been reported in patients with CIIP. ${ }^{23-25}$ Electron microscopy and/or KIT immunolabelling combined with confocal microscopy and image analysis demonstrated a quantitative decrease in ICCs along with structural abnormalities such as loss of processes and damaged intracellular cytoskeleton and organelles. The evidence of significant changes in the ICC enteric network further illustrates the critical role played by these nonneuronal cells in regulating gut motility. ${ }^{26} 27$

\section{Myopathies}

Histopathological analysis of tissue obtained from patients with CIIP due to an underlying primary visceral myopathy showed smooth muscle fibrosis and marked vacuolisation of the circular and longitudinal layers of the intestinal wall, although these changes may predominantly exist in only one layer. $^{711}$ Similar to that described for neuropathies, refinement of morphofunctional techniques (for example, immunohistochemistry and electron microscopy) has significantly improved our understanding of enteric myopathies. ${ }^{28}$ In 1992, Smith and colleagues ${ }^{29}$ described the first case of a specific protein defect with no underlying structural abnormality demonstrable by either light or electron microscopy. In this issue of Gut, Knowles and colleagues ${ }^{30}$ report the results of a multicentre study on a large series of well characterised CIIP patients (studied by small bowel manometry and laparoscopic biopsy) (see page 1583). They showed a selective decrease or absence of $\alpha$-actin staining in the circular muscle of the jejunum in approximately $25 \%$ $(\mathrm{n}=28$ ) of patients with CIIP. Based on these findings, the authors suggested that changes in $\alpha$-actin staining could be used as a biological marker of CIIP.

\section{"Refinement of morphofunctional techniques has signifi- cantly improved our understanding of enteric myopathies"}

Although interesting, these results should be interpreted with caution. Firstly, this study showed that $\alpha$-actin may undergo complex post-translational processing as its expression also appears to be deficient in the control ileum. This raises two questions: one is about the risk of false positive results due to inappropriate sampling during surgery and the other pertains to the specificity of $\alpha$-actin immunoreactivity. Secondly, some of the cases with $\alpha$-actin abnormalities were defined as neurogenic forms of CIIP, thus providing a conceptual gap on the clinical correlate of $\alpha$-actin changes. Thirdly, CIIP is a heterogeneous syndrome and most histopathological features (that is, nerve, muscle, and ICC abnormalities) may co-exist at the tissue level and contribute synergistically to severe dysmotility. Further studies based on $\alpha$-actin assessment with western blotting (to identify conformational changes in the $\alpha$-actin molecule) and/or polymerase chain reaction (to identify possible $\alpha$-actin altered expression) are needed to clarify the meaning of abnormal $\alpha$-actin staining and its association with CIIP.

\section{FUTURE PERSPECTIVES}

Although the pathology of chronic intestinal pseudo-obstruction requires further study before an effective nosology can be definitely proposed, combined clinical and histopathological studies should be encouraged in order to provide the basic framework for standardising the histological evaluation of tissue obtained from patients with CIIP. The growing knowledge in neuropathies, myopathies, and ICC network abnormalities is fundamental for generating new therapeutic approaches. Progress in histopathology will likely help in taking the term "idiopathic" out of intestinal pseudoobstruction syndrome.

\section{ACKNOWLEDGEMENTS}

The original work of the authors was supported by a grant from the Italian Ministry of Education, University and Research (COFIN 2003 to R De G and VS) and funds from the University of Bologna to R De G, RC, and VS. Professor VS was a recipient of a Janssen Foundation Educational grant.

\footnotetext{
Authors' affiliations

R De Giorgio, R Corinaldesi, V Stanghellini, Department of Internal Medicine and Gastroenterology, University of Bologna, Bologna, Italy G Sarnelli, Gastroenterology Unit, Department of Clinical and Experimental Medicine, University "Federico II", Naples, Italy
} 


\section{REFERENCES}

1 Goyal RK, Hirano I. The enteric nervous system. N Engl J Med 1996;334:1106-15

2 Wood JD. Neural and humoral regulation of gastrointestinal motility. In: Schuster MM, Crowell MD, Koch KL, eds. Gastrointestinal motility in health and disease. London: BC Decker, 2002:19-42.

3 Stanghellini V, Corinaldesi R, Barbara L. Pseudo-obstruction syndromes. Baillieres Clin Gastroenterol 1988;2:225-54.

4 Di Lorenzo C. Pseudo-obstruction: Current approaches. Gastroenterology 1999:116:980-7.

5 Coulie B, Camilleri M. Intestinal pseudo-obstruction. Annu Rev Med 1999:50:37-55.

6 De Giorgio R, Guerrini S, Barbara G, et al. New insights into human enteric neuropathies. Neurogastroenterol Motil 2004;16(suppl 1):143-7.

7 De Giorgio R, Camilleri M. Human enteric neuropathies: Morphology and molecular pathology. Neurogastroenterol Motil 2004 (in press)

8 De Giorgio R, Barbara G, Stanghellini V, et al. The pharmacological treatment of acute colonic pseudo-obstruction. Aliment Pharmacol Ther 2001;15:1717-27.

9 Cogliandro R, Stanghellini V, Cogliandro L, et al. Small bowel motility and natural history of chronic intestinal pseudo-obstruction. Gastroenterology 1998;114:A735.

10 Stanghellini V, Camilleri M, Malagelada J-R. Chronic idiopathic intestinal pseudoobstruction: clinical and intestinal manometric findings. Gut 1987;28:5-12.

11 Krishnamurthy S, Schuffler MD. Pathology of neuromuscular disorders of the small intestine and colon. Gastroenterology 1987;93:610-39.

12 Furness JB, Costa M. The enteric nervous system. Edinburgh: Churchill Livingstone, 1987.

13 Gershon MD, Kirchgessner AL, Wade PR. Functional anatomy of the enteric nervous system. In: Johnson LR, ed. Physiology of the gastrointestinal tract. New York: Raven Press, 1994:381-422.

14 De Giorgio R, Guerrini S, Barbara G, et al. Inflammatory neuropathies of the enteric nervous system. Gastroenterology 2004;126:1872-83.

15 Smith VV, Gregson N, Foggensteiner L, et al. Acquired intestinal aganglionosis and circulating autoantibodies without neoplasia or other neural involvement. Gastroenterology 1997;112:1366-71.

16 De Giorgio R, Barbara G, Stanghellini V, et al. Idiopathic myenteric ganglionitis underlying intractable vomiting in a young adult. Eur J Gastroenterol Hepatol 2000;12:613-16.
17 De Giorgio R, Barbara G, Stanghellini V, et al. Clinical and morphofunctional features of idiopathic myenteric ganglionitis underlying severe intestinal motor dysfunction: a study of three cases. Am J Gastroenterol 2002;97:2454-9.

18 King PH, Redden D, Palmgren JS, et al. Hu antigen specificities of ANNA-I autoantibodies in paraneoplastic neurological disease. J Autoimmun 1999; 13:435-43.

19 Schäppi MG, Smith VV, Milla PJ, et al. Eosinophilic myenteric ganglionitis is associated with functional intestinal obstruction. Gut 2003;52:752-5.

20 Hall KE, Wiley JW. Neural injury, repair and adaptation in the GI tract. I. New insights into neuronal injury: a cautionary tale. Am J Physiol 1998;274:G978-83.

21 Hockenbery D, Nunez G, Milliman C, et al. Bcl-2 is an inner mitochondrial membrane protein that blocks programmed cell death. Nature 1990:348:334-6.

22 De Giorgio R, Barbara G, Stanghellini V, et al. Reduced bcl-2 expression in the enteric nervous system (ENS) as a marker for neural degeneration in patients with gastrointestinal motor disorders (GIMD). Gastroenterology 2000; 118:A4821

23 Isozaki K, Hirota S, Miyagawa J-I, et al. Deficiency of c-kit+ cells in patients with a myopathic form of chronic idiopathic intestinal pseudo-obstruction. Am J Gastroenterol 1997;92:332-4.

24 Boeckxstaens GE, Rumessen JJ, de Wit L, et al. Abnormal distribution of the interstitial cells of Cajal in an adult patient with pseudo-obstruction and megaduodenum. Am J Gastroenterol 2002:97:2120-6.

25 Feldstein AE, Miller SM, El-Youssef M, et al. Chronic intestinal pseudoobstruction associated with altered interstitial cells of Cajal network. J Pediatr Gastroenterol Nutr 2003;36:492-7.

26 Huizinga JD, Thuneberg L, Vanderwinden JM, et al. Interstitial cells of Cajal as targets for pharmacological intervention in gastrointestinal motor disorders. Trends Pharmacol Sci 1997; 18:393-403.

27 Sanders KM, Ördög T, Ward SM. Physiology and pathophysiology of the interstitial cells of Cajal: from bench to bedside. IV. Genetic and animal models of $\mathrm{Gl}$ motility disorders caused by loss of interstitial cells of Cajal. Am J Physiol 2002;282:G747-56.

28 Moore SW, Schneider JW, Kaschula ROC. Unusual variations of gastrointestinal smooth muscle abnormalities associated with chronic intestinal pseudoobstruction. Pediatr Surg Int 2002;18:13-20.

29 Smith VV, Lake BD, Kamm MA, et al. Intestinal pseudo-obstruction with deficient smooth muscle alpha-actin. Histopathology 1992;21:535-42.

30 Knowles CH, Silk DBA, Darzi A, et al. Deranged smooth muscle $\alpha$-actin as a biomarker of intestinal pseudo-obstruction: a controlled multinational case series. Gut 2004;53:1583-9.

\section{EDITOR'S QUIZ: GI SNAPSHOT}

\section{Unusual complication of tuberous sclerosis complex}

\section{Clinical presentation}

A 39 year old man with a known history of tuberous sclerosis complex (TSC) presented with haematuria due to haemorrhagic changes of a large renal angiomyolipoma. Selective transcatheter arterial embolisation of the lesion was performed. Haematuria resolved but the patient experienced postembolisation syndrome with fever, and presented with bowel obstructive symptoms. Abdominal radiographs showed marked dilatation of the colon. An acute colonic pseudoobstruction (Ogilvie's syndrome) was suspected and colonoscopic decompression was performed. Colonoscopy showed dilatation of the colon without mechanical obstruction and multiple sessile polyps localised in the rectum (fig lA). One polyp was biopsied and fig $1 \mathrm{~B}$ shows the microscopic features.

\section{Question}

What is the diagnosis?

See page 1565 for answer

This case is submitted by:

D Chatelain, H Sevestre

Department of Pathology, CHU Amiens, Amiens Cedex 01, France H Zaher, M Flamant, F Brazier, G Geslin, J-L Dupas Department of Gastroenterology, CHU Amiens, Amiens Cedex 01, France J-M Regimbeau Department of Surgery, CHU Amiens, Amiens Cedex 01, France

Correspondence to: D Chatelain, Service d'Anatomie Pathologique, $\mathrm{CHU}$ Amiens, Place Victor Pauchet, 80054 Amiens Cedex 01, France Chatelain.denis@chu-amiens.fr

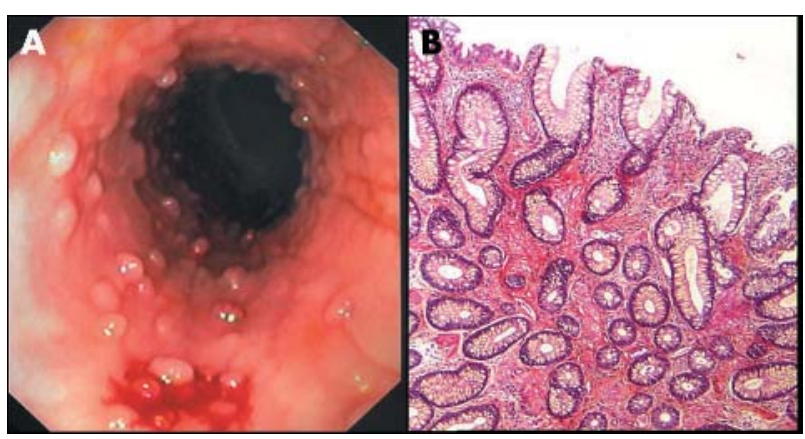

Figure 1 (A) Endoscopy of the colon. (B) Microscopic examination of a biopsied polyp 